\title{
Cultural bias and the retention and organization of verbal information: \\ A developmental perspective
}

\author{
Paul Miller, Nancy Joubran-Awadie, Raphiq Ibrahim* \\ Department of Special Education, University of Haifa \\ mpaul@edu.haifa.ac.il \\ Learning Disabilities Department, University of Haifa \\ nancyjoub@gmail.com
}

The Edmond J. Safra Brain Research Center and the Learning Disabilities Department, University of Haifa raphiq@psy.haifa.ac.il

\begin{abstract}
This study explores how cultural disposition and education impact the ways individuals assimilate and organize information. Students from two cultural backgrounds (individualist, collectivist) and three levels of education (elementary school, high school and university) were tested in two experiments. Findings from Experiment 1, which used a short-term memory (STM) paradigm, revealed that, contrary to a widely held theory, culture per se does not seem to foster propensities toward the use of particular memory strategies. Experiment 2, which used a concept organization paradigm, suggests that, even if cultural/educational preferences bias individuals' approach to information in some regards, such bias is overshadowed by and interacts with other more intuitive predispositions. Findings are discussed from a cultural and educational point of view.
\end{abstract}

\section{Indexing terms/Keywords}

Culture; education; memor; organization; concept

\section{Academic Discipline And Sub-Disciplines}

CULTURE; MEMORY; VERBAL INFORMATION

\section{Council for Innovative Research}

Peer Review Research Publishing System

Journal: Journal of Advances in Linguistics

Vol. 5, No. 2

editorjalonline@gmail.com

www.cirjal.com 


\section{INTRODUCTION}

In order to optimize adjustment to an increasing complex world, individuals are compelled to develop strategies that allow them to organize information in economical ways (Miller \& Eilam, 2008; Nguyen \& Murphy, 2003). According to some social psychologists, objects can be organized based upon their taxonomic relationship or their thematic relationship (Ji, Zhang, \& Nisbett, 2004; Coley, Vitkin, Seaton, \& Yopchick, 2005). Thematic relationships reflect associations that develop following the recurrence of spatially coinciding or temporally continuous phenomena (e.g., flower and bee, lightning and thunder) (Ji, Zhang, \& Nisbett, 2004; Masuda \& Nisbett, 2001; Miller \& Eilam, 2008). Given that such relations are readily observed and intuitively perceived and remembered, researchers assume that knowledge of them determines and structures individuals' behavior from a relatively early age (Baldwin, 1992; Gelman, 1998; Markman \& Hutchinson, 1984; Micklo, 1995; Nguyen \& Murphy, 2003). Thematic relationships reflect associations that develop following the recurrence of spatially coinciding or temporally continuous phenomena (e.g., flower and bee, lightning and thunder) ( $\mathrm{Ji}$, Zhang, \& Nisbett, 2004; Masuda \& Nisbett, 2001; Miller \& Eilam, 2008). Given that such relations are readily observed and intuitively perceived and remembered, researchers assume that knowledge of them determines and structures individuals' behavior from a relatively early age (Baldwin, 1992; Gelman, 1998; Markman \& Hutchinson, 1984; Micklo, 1995; Nguyen \& Murphy, 2003).

Taxonomic organization is based on the idea that objects share causal, spatial and temporal relationships (Markham \& Hutchinson, 1984). Such taxonomic organizations - often referred to as categories - are based upon a critical mass of overlapping concrete and/or abstract features of particular phenomena (Anderson, 1991; Klausmeier, 1980; Pennequin, Fontaine, Bonthoux, Scheuner, \& Blaye, 2006). For example, sparrow, eagle and hawk all share properties of the category "bird," in conjunction with some further distinguishing properties (Murphy, 2002). As a consequence, the density of information that has to be stored for each category member is considerably reduced, which, in turn, is assumed to economize its storage, facilitate its retrieval for future application and enhance the development of further knowledge (Baldwin, 1992; Markman, 1989)

Some studies have pointed to a shift from the use of thematic organization to more complex organization strategies in the course of maturation (Blewitt, 1994; Denney \& Ziobrowski, 1972; Inhelder \& Piaget, 1964; Kofsky, 1966; Lowell, 1980). More recent evidence, however, suggests that thematic relations continue to play an important role in the organization of information throughout life (Borghi \& Caramelli, 2003; Miller \& Eilam, 2008; Murphy, 2002; Lin \& Murphy, 2001). Moreover, there is growing evidence that particularities of the individual's cultural background have a marked impact on the quality of recollected information (Gutchess \& Indeck, 2009; Koutstaal, Wanger, Rotte, Maril, Buckner, \& Schacter, 2001; Medin \& Atran, 2004; Nisbett, Peng, Choi, \& Norenzayan, 2001; Wang \& Ross, 2010). It is in particular the imparity between values transferred by collectivist as opposed to individualist cultures that has attracted scholarly attention (Hofstede \& Bond, 1984; Triandis, 1995).

Some evidence suggests that collectivists tend to stress the importance of the collective over the individual, with particular emphasis on contextual and relational aspects between items rather than their individual properties (e.g., Masuda \& Nisbett, 2001; Nisbett, Peng, Choi, \& Norenzayan, 2001). In contrast, individualists seem to advocate independence, individuality and self-reliance as the central life experience, with attention being tuned to individual characteristics of objects and events rather than to the context and relationships in which they appear (e.g., Masuda \& Nisbett, 2001; Nisbett, Peng, Choi, \& Norenzayan, 2001). Such differences in cultural disposition are likely to modify the way individuals structure information and knowledge in their memory.

Another possibility is that differences in organization preferences (e.g., taxonomic vs. thematic) at all age levels may originate first and foremost from the desire to utilize strategies that optimally help the individual perform specific tasks (Denney \& Ziobrowski, 1972; Kalish \& Gelman, 1992). According to this position, the ability to apply different organization strategies is a prerequisite for an adequate functioning of individuals in their surroundings (Medin, Coley, Storms, \& Hayes, 2003; Nguyen \& Murphy, 2003). The tendency of children to use thematic organization strategies may, therefore, simply reflect the fact that such strategies best fit their needs, rather than indicating an inability to use other organization criteria as well (Miller \& Eilam, 2008).

In sum, evidence suggests that individuals' maturation and cultural background play a determining role in the way they organize and represent their experience in permanent memory. People growing up in individualist cultures seem to prefer thinking in taxonomic categories (e.g., Gutchess \& Indeck, 2009). In contrast, people from collectivist cultures have been claimed to exhibit a propensity to stress thematic (contextual) relations when assimilating information (e.g., Gutchess \& Indeck, 2009; Kitayama, Duffy, Kawamura, \& Larsen, 2003).

\section{THE PRESENT STUDY}

The present study aims to further clarify culture-related cognitive preferences by comparing samples of typical urban Israeli Jews and a typical urban Israeli Arabs from different levels of education population, with the former being assumed to manifest a more individualist and the latter a more collectivist life orientation (e.g., Diab \& Mi'ari, 2007; Mahajna, Benzion, Bogaire, \& Shavit, 2007; Mikulicer, Weller, \& Florian, 1993; Sabbagh, Faher-Aladeen, \& Resh, 2004; Sagy, Orr, Bar-On, \& Awwad, 2001). Participants were tested with a short-term memory (STM) paradigm that manipulated to-berecalled word lists according to different structural criteria (thematic, taxonomic, unstructured) and a concept organization paradigm that required participants to organize randomly distributed written word concepts into clusters that make sense. Note, the former requires the retention and recall of prestructured information in a primarily passive manner, whereas the latter requires active application of structural knowledge to organize randomly presented word concepts. 


\subsection{Research Question and Hypotheses}

The central question of the present study was whether culture and schooling bias the formation of knowledge structures that underlie individuals' assimilation and organization of information. We tested the following hypotheses:

1. Both Israeli Jewish and Israeli Arab participants will manifest greater STM recall for structured word lists than unstructured lists.

2. Jewish participants will manifest better STM recall for word lists structured taxonomically than for the same word lists structured thematically. In contrast, Arab participants who will manifest better recall for lists structured thematically than taxonomically.

3. Jewish participants will organize word concepts predominantly into clusters reflecting a taxonomic relationship, whereas Arab participants will cluster items primarily based on thematic relations.

4. The impact of cultural preferences on STM recall and concept organization will increase with prolonged exposure to the culture.

In general, the above hypotheses are motivated by a theoretical orientation assuming that differences in how individuals process information reflect educational and culture-specific expertise rather than a maturation-driven shift in their cognitive abilities (Carey, 1985; Gelman \& Kalish, 2006).

\section{EXPERIMENT 1: STM PARADIGM}

Experiment 1 examined whether cultural orientation and formal education impact participants' ability to temporarily retain information for immediate written recall, a primarily passive task.

\subsection{Method}

\subsubsection{Participants}

Two samples (60 Israeli Jews and 60 Israeli Arabs) were equally divided into three age groups: elementary school, high school and university (Table 1). The elementary and high school students were recruited from schools that serve a population of an average socioeconomic background (SES data were obtained from the Ministry of Education). The university students were recruited via a flyer distributed on campus.

Table 1. Participant Samples

\begin{tabular}{|c|c|c|c|c|c|c|c|c|}
\hline \multicolumn{9}{|l|}{ Level of Formal } \\
\hline \multirow[t]{2}{*}{ Education } & \multicolumn{4}{|c|}{ Jewish } & \multicolumn{4}{|c|}{ Arab } \\
\hline & Female & Male & Age & $\mathrm{n}$ & Female & Male & Age & $\mathrm{n}$ \\
\hline $4^{\text {th }}-5^{\text {th }}$ grade & 12 & 8 & $9-11$ & 20 & 10 & 10 & $9-11$ & 20 \\
\hline $10^{\text {th }}-11^{\text {th }}$ grade & 11 & 9 & $15-16$ & 20 & 10 & 10 & $15-16$ & 20 \\
\hline University students & 12 & 8 & $21-35$ & 20 & 11 & 9 & $20-26$ & 20 \\
\hline
\end{tabular}

Jewish participants all spoke Hebrew as their native language, a language they also used for reading and writing. They were raised in a cultural environment emphasizing individualist values. Arab participants used spoken Arabic as their native language and Modern Standard Arabic (MSA) for reading and writing. Both spoken Arabic and MSA are also used in school to teach the curriculum. Arab participants were raised in a cultural environment stressing primarily collectivist values.

All participants had normal hearing and normal or corrected-to-normal visual skills. Only participants with no record of learning disabilities, attention disorders or behavioral disorders were included in the sample.

\subsubsection{Material and stimuli}

The STM paradigm used 12 word lists. Each list included nine familiar nouns presented to participants in consecutive order on a computer display.

The items in each of the nine-word lists were divided into three-word triads that allowed for the preparation of three presentation modes (Table 2): a) each triad presented taxonomically-related items; b) each triad presented thematicallyrelated items; and c) the nine words in the list were presented in an unstructured manner (randomly mixed). Two additional nine-word lists were prepared for task explanation and practice.

\subsubsection{Procedure}

Participants were examined individually in a quiet place at their school, university or home. Students from elementary and high school were tested only after consent had been obtained from the relevant agencies and their parents. 
The STM paradigm was explained to participants in their native tongue. Participants were asked to sit in front of a laptop. They were told that they would see lists of words appearing one after another in the center of the display and were instructed to write them down on an empty booklet page the moment a string of four stars (instead of a word) appeared. Two warm-up word lists were given to ensure understanding of the task instructions.

The 12 nine-word lists were rotated in four blocks, with each block containing three presentations. Within each block, the three lists were rotated according to the three presentation modes (taxonomically structured, thematically structured, unstructured). As a result, all stimulus lists were presented in all three presentation modes, which counterbalanced for possible bias from variation in vocabulary on the recall of items presented in each mode. Moreover, to counterbalance for practice and fatigue, different participants saw the stimulus lists at different positions within the entire presentation of the 12 stimulus lists. Stimulus lists were presented one after another, with recall for each of them written down on a new blank page for future analysis.

Table 2. Example of a nine-word list in Hebrew and Arabic according to three modes of presentation

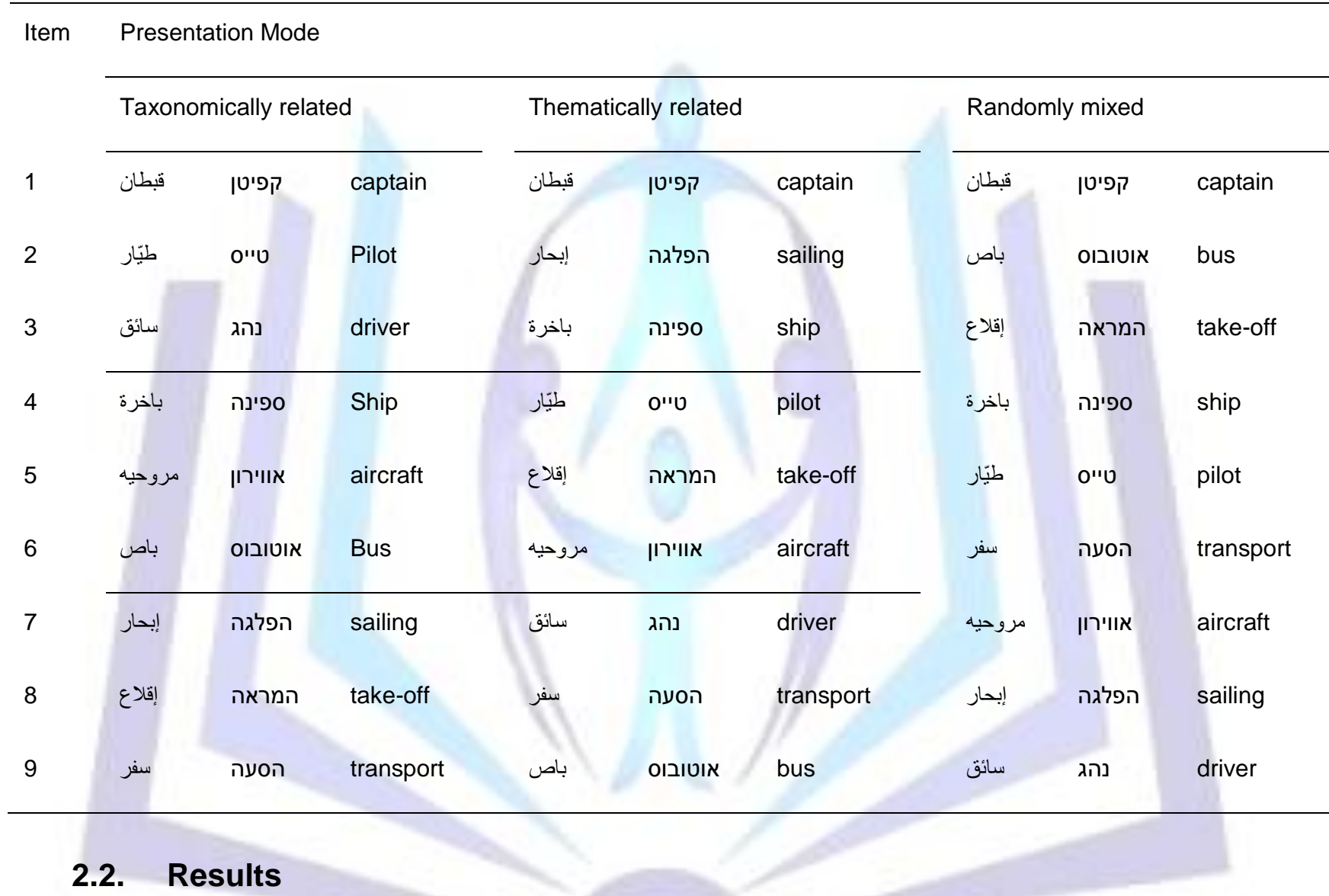

We conducted three analyses of variance (ANOVA), one analyzing number of recalled items, one recall-order accuracy, and one intrusion error rates. In all of them, presentation mode (taxonomic, thematic, unstructured) was computed as a within-subject factor and cultural background (individualist, collectivist) and grade-level (elementary school, high school, university) as two between-subject factors.

\subsubsection{Number of recalled items}

For each participant, recall per list was scored on a scale from 0-9. Mean recall rates, per presentation mode, participants' cultural background and grade-level are presented in Table 3.

Cultural background produced a statistically significant main effect, $F(1,114)=5.42, p<.05, \eta^{2}=.05$, indicating that, on the average, Jewish participants recalled more test items than Arab participants. Grade-level was also statistically significant, $F(2,114)=78.10, p<.001, \eta^{2}=.58$, i.e., schooling impacted the number of recalled items. The interaction between cultural background and grade-level was statistically not significant, $F(2,114)=1.18$, suggesting that schooling impacted Arab and Jewish participants uniformly. We conducted Tukey post hoc analysis to clarify the final significance of the grade-level main effect. Findings indicated that recall increased proportionally with ascending grade-level for both Jewish and Arab participants (all p's <.05).

Mode of stimulus presentation produced a statistically significant main effect, $F(1,114)=57.01, p<.001, \eta^{2}=.33$, implying that the number of correctly recalled items was not the same for the three presentation modes ( Table 3 ). 
Presentation mode did significantly interacted with the participants' cultural background, $F(1,114)=.03$, or grade-level, $F(2,114)=1.54$.

To clarify the final significance of the presentation mode main effect, we used three paired post hoc $t$-tests (two-tailed) to compare the amount of recalled items in the three presentation modes. Recall for unstructured stimulus lists was significantly poorer than for thematically or taxonomically structured lists, $t(119)=-8.00, p<.001 ; t(119)=-7.58, p<.001$, respectively. There was no significant difference between thematically and taxonomically-related lists.

Table 3. Average number of recalled items per presentation mode, culture and grade level (standard deviations in parentheses)

\begin{tabular}{|c|c|c|c|c|}
\hline Presentation Mode & Elementary School & High School & University & All Levels \\
\hline & \multicolumn{3}{|c|}{ Jewish Participants - Individualist Background } & \\
\hline Taxonomic & $4.90(1.46)$ & $6.65(1.20)$ & $7.56(1.13)$ & $6.37(1.67)$ \\
\hline Thematic & $5.13(1.56)$ & $6.78(1.55)$ & $7.71(0.92)$ & $6.54(1.73)$ \\
\hline Unstructured & $4.35(1.11)$ & $5.89(1.27)$ & $6.75(1.05)$ & $5.66(1.51)$ \\
\hline \multirow[t]{2}{*}{ All Lists } & $4.79(1.18)$ & $6.44(1.19)$ & $7.34(0.94)$ & $6.19(1.52)$ \\
\hline & \multicolumn{3}{|c|}{ Arab Participants - Collectivist Background } & \\
\hline Taxonomic & $4.36(1.25)$ & $6.73(0.94)$ & $6.86(1.07)$ & $5.98(1.58)$ \\
\hline Thematic & $4.23(1.32)$ & $6.83(1.11)$ & $7.31(0.81)$ & $6.12(1.74)$ \\
\hline Unstructured & $3.91(0.87)$ & $5.68(0.82)$ & $6.14(1.34)$ & $5.24(1.40)$ \\
\hline \multirow[t]{2}{*}{ All Lists } & $4.17(0.76)$ & $6.41(0.62)$ & $6.77(0.94)$ & 5.78 (1.39) \\
\hline & All Participants & & & \\
\hline Taxonomic & $4.63(1.37)$ & $6.69(1.06)$ & $7.21(1.14)$ & $6.18(1.63)$ \\
\hline Thematic & $4.68(1.49)$ & $6.80(1.33)$ & $7.51(0.88)$ & $6.33(1.74)$ \\
\hline Unstructured & $4.13(1.01)$ & $5.78(1.06)$ & $6.44(1.23)$ & $5.45(1.47)$ \\
\hline All Lists & $4.48(1.03)$ & $6.42(0.93)$ & $7.06(0.97)$ & $5.99(1.47)$ \\
\hline
\end{tabular}

\subsubsection{Recall-order accuracy}

Recall-order accuracy reflects the degree to which item recall order matched stimulus presentation order. This variable was calculated as a percentile factor relative to the total number of words recalled from a particular eight-word list. ${ }^{i}$ Table 4 shows average percentile recall-order accuracy for the three presentation modes, with reference to cultural background and grade-level.

Cultural background produced a statistically significant main effect, $F(1,114)=12.52, p=.001, \eta^{2}=.10$, indicating that, on the average, Jewish participants retained item presentation order better than Arab counterparts. The main effect of gradelevel was also statistically significant, $F(2,114)=13.34, p<.001, \eta^{2}=.19$, indicating that the ability to retain item order was markedly impacted by schooling. The interaction between cultural background and grade-level failed to reach statistical significance, $F(2,114)=.59$, suggesting that schooling impacted recall-order accuracy uniformly for Arab and Jewish participants.

We used Tukey post hoc procedure to clarify the final significance of the grade-level main effect on recall-order accuracy. Elementary school students were found to have markedly poorer retention of item presentation order than high school and university students, all $p$ 's $\leq .05$. High school and university students did not differed in this regard.

Presentation mode significantly impacted accuracy of item presentation order, $F(2,114)=33.23, p<.001, \eta^{2}=.23$. Paired post hoc $t$-tests (two-tailed) clarifying the presentation mode effect pointed to markedly increase accuracy for thematically or taxonomically structured item lists than for unstructured lists, $t(119)=6.78, p<.001 ; t(119)=6.70, p<.001$, respectively. Thematically and taxonomically presented word lists did not differ in this regard. 
Table 4. Average percentile recall-order accuracy per presentation mode, culture and grade level (standard deviations in parentheses)

\begin{tabular}{|c|c|c|c|c|}
\hline Presentation Mode & Elementary School & High School & University & All Levels \\
\hline & \multicolumn{3}{|c|}{ Jewish Participants - Individualist Background } & \\
\hline Taxonomic & $69.65(16.03)$ & $79.53(13.75)$ & $83.35(11.13)$ & $77.51(14.75)$ \\
\hline Thematic & $69.04(16.19)$ & $81.03(12.30)$ & $85.44(9.63)$ & $78.50(14.55)$ \\
\hline Unstructured & $63.51(12.40)$ & $72.10(8.91)$ & $66.20(11.55)$ & $67.27(11.45)$ \\
\hline \multirow[t]{2}{*}{ All Lists } & $67.40(12.49)$ & 77.55 ( 8.27) & 78.33 ( 8.67) & $74.43(11.03)$ \\
\hline & \multicolumn{3}{|c|}{ Arab Participants - Collectivist Background } & \\
\hline Taxonomic & $64.31(14.99)$ & $66.96(17.28)$ & $77.16(14.93)$ & $69.48(16.48)$ \\
\hline Thematic & $58.28(17.65)$ & $75.05(15.45)$ & $78.99(11.06)$ & $70.77(17.28)$ \\
\hline Unstructured & $59.65(15.79)$ & $61.66(11.84)$ & 65.35 ( 9.44) & $62.22(12.64)$ \\
\hline \multirow[t]{2}{*}{ All Lists } & $60.74(12.41)$ & $67.89(12.50)$ & 73.83 ( 8.98) & $67.49(12.45)$ \\
\hline & All Participants & & & \\
\hline Taxonomic & $66.98(15.56)$ & $73.24(16.68)$ & 80.25 (13.37) & 73.49 (16.08) \\
\hline Thematic & $63.66(17.58)$ & $78.04(14.11)$ & $82.21(10.74)$ & 74.64 (16.37) \\
\hline Unstructured & $61.58(14.15)$ & $66.88(11.62)$ & $65.77(10.41)$ & $64.74(12.28)$ \\
\hline All Lists & $64.07(12.74)$ & $72.72(11.55)$ & $76.08(9.00)$ & 70.96 (12.22) \\
\hline
\end{tabular}

Cultural background was not found to interact with presentation mode, $F(2,114)=.77$, but interacted significantly with grade-level, $F(4,114)=5.96, p<.001, \eta^{2}=.10$, suggesting schooling to impact students' ability to retain item order. The three-way interaction presentation mode $x$ cultural background $x$ grade-level was not significant, $F(4,114)=1.25$, implying that variance from grade-level was similar for Jewish and Arab participants.

We conducted a series of post hoc Oneway analyses of variance to clarify the final significance of the presentation mode $x$ grade-level interaction. Results suggest that elementary school participants had better retention of presentation order for taxonomically structured word lists than for unstructured ones, $t(39)=2.77, p<.01$, while no significant difference was found between thematically structured word lists, on the one hand, and taxonomically structured or unstructured word lists, on the other. For high school students, retention of stimulus presentation order for thematically or taxonomically structured lists was markedly better than for unstructured lists, $t(39)=4.72, p<.001 ; t(39)=2.74, p<.01$, respectively. Moreover, they retained presentation order better for thematically than for taxonomically structured lists, $t(39)=2.17, p<.05$. Finally, university students exhibited significantly better retention of presentation order for thematically and taxonomically structured than unstructured stimulus lists, $t(39)=9.41, p<.001 ; t(39)=6.38, p<.001$, respectively. Taxonomically structured and thematically structured lists did not differ in this regard.

\subsubsection{Intrusion error rates}

Intrusion errors were words jotted down by participants during recall of a particular list that did not belong to that list. Table 5 presents average intrusion error rates for the three presentation modes, by participants' cultural background and gradelevel.

Cultural background did not produce a statistically significant main effect, $F(1,114)=.07$, pointing to comparable intrusion error rates for Jewish and Arab participants. Nor was the grade-level main effect statistically significant, $F(2,114)=.06$, suggesting that, overall, intrusion error rates were similar regardless of schooling level. The lack of a statistically significant interaction between cultural background and grade-level, $F(2,114)=1.53$, suggested that this to be true for Jewish and Arab participants alike. 
Table 5. Average intrusion error rates per stimulus list, by presentation mode, culture and grade level (standard deviations in parentheses)

\begin{tabular}{|c|c|c|c|c|}
\hline Presentation Mode & Elementary School & High School & University & All Levels \\
\hline & \multicolumn{3}{|c|}{ Jewish Participants - Individualist Background } & \\
\hline Taxonomic & $.54(.42)$ & $.53(.67)$ & $.29(.41)$ & $.45(.52)$ \\
\hline Thematic & $.28(.34)$ & $.31(.38)$ & $.40(.37)$ & $.33(.36)$ \\
\hline Unstructured & $.54(.42)$ & $.36(.36)$ & $.56(.57)$ & $.33(.36)$ \\
\hline \multirow[t]{2}{*}{ All Lists } & $.45(.32)$ & $.40(.35)$ & $.34(.25)$ & $.40(.31)$ \\
\hline & \multicolumn{3}{|c|}{ Arab Participants - Collectivist Background } & \\
\hline Taxonomic & $.45(.32)$ & $.40(.35)$ & $.34(.25)$ & $.40(.31)$ \\
\hline Thematic & $.31(.31)$ & $.33(.40)$ & $.39(.45)$ & $.34(.39)$ \\
\hline Unstructured & $.40(.39)$ & $.45(.39)$ & $.33(.32)$ & $.47(.46)$ \\
\hline \multirow[t]{2}{*}{ All Lists } & $.37(.23)$ & $.38(.18)$ & $.49(.44)$ & $.41(.30)$ \\
\hline & \multicolumn{3}{|l|}{ All Participants } & \\
\hline Taxonomic & $.46(.41)$ & $.44(.51)$ & $.40(.47)$ & $.44(.44)$ \\
\hline Thematic & $.29(.32)$ & $.32(.38)$ & $.39(.40)$ & $.33(.37)$ \\
\hline Unstructured & $.47(.41)$ & $.41(.37)$ & $.44(.47)$ & $.44(.42)$ \\
\hline All Lists & $.41(.28)$ & $.39(.28)$ & $.41(.36)$ & $.40(.31)$ \\
\hline
\end{tabular}

The main effect of presentation mode was statistically significant, $F(2,114)=3.42, p<.05, \eta^{2}=.03$, implying that intrusion error rates were biased by mode of presentation. Three paired post hoc $t$-tests (two-tailed) comparing intrusion error rates between presentation modes revealed significantly reduced intrusion error rates for thematically structured stimulus lists compared to unstructured ones, $t(119)=2.44, p<.05$, and taxonomically structured ones, $t(119)=-2.18, p<.05$. Unstructured and taxonomically structured stimulus lists did not differed in this regard.

\subsection{Discussion}

The basic assumption of this paradigm was that presenting information in a structured way benefits its assimilation into prestructured knowledge representations and, consequently, facilitates its retrieval and application later on (Medin, Coley, Storms, \& Hayes, 2003; Nguyen \& Murphy, 2003). Based on this assumption, we expected both Israeli Jewish and Israeli Arab participants to manifest better STM recall for structured word lists than for unstructured ones (Hypothesis 1). Findings fully supported this hypothesis. Particularly with regard to the number of recalled items, presenting structured stimulus items proved highly beneficial regardless of cultural background and level of formal education. Similar gains from structure were evident with regard to recall-order accuracy and the appearance of intrusion errors, although thematic structuring seemed to sustain this type of information somewhat better than taxonomic structuring.

From the evidence above, it is obvious that the human brain assimilates perceived information by means of structured knowledge. In instances where such information is structured in a way that overlaps with existing knowledge structures, its processing becomes notably enhanced. The central question this experiment sought to answer was does the formation of knowledge structures reflect education and culture-specific expertise (Carey, 1985; Gelman \& Kalish, 2006)?

We expected to receive an answer to this question by testing whether participants from an Israeli Jewish background (individualist cultures) are superior in recalling taxonomically structured word lists as opposed to the same word lists presented thematically structured, as well as whether participants from an Israeli Arab background (collectivist cultures) showed an opposite performance profile, with thematically structured word lists recalled better than taxonomically structured ones (Hypothesis 2). Surprisingly, analysis of our data highlighted similarities rather than differences between the two cultures. Recall following the two prestructured presentation modes was not significantly biased by the participants' cultural background and was, as already stated, markedly better than recall following unstructured word lists for both Jewish and Arab participants. Taken together, these findings challenge previous research conclusions that cultural differences modify the recall of taxonomically versus thematically-related information (e.g., Masuda \& Nisbett, 2001; Nisbett, Peng, Choi, \& Norenzayan, 2001; Wang \& Ross, 2005).

The absence of cultural bias on the participants' recall performance in this experiment suggests that the impact of cultural preference on STM performance may have been overstated. It seems that presentation mode, rather than cultural background, is an essential factor in determining individuals' ability to retain information. In support of this conclusion is the finding of highly beneficial effects on recall originating from the presentation of information structured - both taxonomically and thematically - as opposed to unstructured. This was found to be true for both Jewish and Arab participants at all grade-levels, as predicted by our first hypothesis. 
The above findings emphasize the fundamental impact of organization on the individual's cognitive processing, a contribution that has also been revealed in many other studies (e.g., Miller \& Eilam, 2008; Poirier, Dhir, Saint-Aubin, Tehan, \&, Hampton, 2011; Poirier \& Saint-Aubin, 1995, 1999; Vicari, Pasqualetti, Marotta, \& Carlesimo, 1999). The fact that our STM paradigm pinpointed such structure-related enhancement in relation to both thematically and taxonomically structured lists with the same stimulus words is noteworthy, as it suggests that perceived information is encoded simultaneously into essentially different knowledge structures, each of which independently sustains the individual's ability to access it for future use.

Another hypothesis tested was that prolonged exposure to a particular culture would produce gains in the recall of word lists that were structured according to cultural preferences (Hypothesis 4). Assuming this to be true, we expected the impact of cultural preferences to be stronger among participants from higher than lower grade-levels. However, the findings seem to refute that assumption. In fact, the linear and uniform improvement in recall performance from one gradelevel to the next, observed for both Jewish and Arab participants, regardless of presentation mode (thematic, taxonomic unstructured), strongly suggests that the capacity to perceive and retain information is not notably modified by the individual's cultural predisposition, but rather seems to be primarily and intrinsically linked to their education and/or maturation level (Cowan et al., 2010; Vicari, Pasqualetti, Marotta, \& Carlesimo, 1999).

Evidence concerning development in amount of recall - as opposed to retention of recall order - does not point to bias from the participants' cultural background. It does, however, suggest that these two abilities develop independently and that their development is affected differently by maturation and/or education. In other words, it seems that the amount of recall is an ability that improves with maturation and thus occurs whether information is structured or not (taxonomic, thematic, unstructured). In contrast, the process of retaining information order appears to reflect an improvement in the use of structured information; this is obvious because gains in this regard were found only in relation to the recall of structured information (taxonomic and/or thematic item lists) but not unstructured information. Moreover, this improvement was not found beyond high school level, suggesting that the ability to use structure to enhance the retention of order information stagnates at a level (76\% accuracy) far from being optimal, a finding that seems to have educational implications. Whether this stagnation reflects a failure of education to explicitly foster learning strategies that stress the vital role of structure in the comprehension of information cannot be determined by this experiment, but it should be considered a possibility worthy of further investigation.

A closer look at the significant interaction between presentation mode and grade-level reveals a rather complex picture regarding the impact of presentation mode on recall-order accuracy, overall and for each of the three levels of schooling. It is noteworthy that at the elementary school level, recall-order accuracy was significantly facilitated by taxonomically structured item presentation, but not by thematically structured item presentation, whereas at the higher grade-levels, both types of presentation structures notably benefited the retention of word presentation order. Moreover, for high school students, thematically structured stimulus lists seemed to facilitate the retention of item presentation order more effectively than taxonomically structured lists, while both presentation structures were equally beneficial for university students. These findings, together with the fact that elementary school students manifested a generally poorer ability to retain word presentation order, lead to the conclusion that the ability to use structure effectively to enhance the retention of information is, by and large, developmentally determined.

Interestingly, equal gains from both taxonomically and thematically organized information were found only for university students, whereas at the high school level, participants were less successful in recruiting taxonomic structure as a prop for retaining word presentation order. This suggests that thematic relations are more intuitively perceived and consequently more accessible as a means for the retention of order information than taxonomic relations, which are abstract representations that cannot be experienced directly.

Intrusion error rates were overall rather small. Although we did not formulate specific hypotheses regarding the occurrence of intrusion errors and the way they interact with presentation modes and cultural background at different grade-levels, their analysis nevertheless revealed some interesting findings. First and foremost is that intrusion errors were mainly determined by presentation mode, with lower rates found for thematically structured presentations than for taxonomically structured or unstructured ones. This was true for all participants, regardless of cultural background. A position proposed in literature (e.g., Tse, 2009) suggests related word lists to produce higher intrusion error rates than unrelated lists. This is because of an increased probability of related words to trigger associations with information not presented. Our findings that thematically structured word lists produced markedly smaller intrusion errors than taxonomically structured or unstructured ones - with no difference between the latter pair - warrant a different explanation. More specifically, it seems that more intuitive relationships between pieces of information, such as thematic relations, leave less space for the associative interference that underlies the occurrence of intrusion errors.

An interesting - albeit incidental - finding of this experiment was that Jewish participants manifested a consistent advantage over Arab participants in recall performance, regardless of the presentation mode and whether amount of recal or recall-order accuracy were considered. A possible explanation for this apparent advantage of the Jewish participants may be rooted in orthography-inherent differences between Hebrew and Arabic. For example, recent studies suggest reading of Arabic texts to be particularly challenging at the orthographic level due to markedly enhanced visual confusability between MSA letter graphemes (e.g., Ibrahim, 2010; Ibrahim, Eviatar, \& Aharon-Peretz, 2002; Miller \& Peleg, 2010).

Moreover, Arabic script may also be more demanding when writing it. Given that the experiment required written recall, it may well be that increased attention to the writing process - in addition to the word encoding process - further reduced recall performance among Arab participants (Barrouillet, Bernardin, Portrat, Vergauwe, \& Camos, 2007). Finally, Arab 
readers are forced to read in a diaglossic context, where there is notable distance between the spoken dialect and the written literary language. As a consequence, Arab participants probably had experience with the stimulus words primarily in their written form (Ibrahim, 2010), whereas Hebrew readers were probably exposed to these words in both their spoken and written forms and, as a consequence, could internalize them to a deeper degree. Taken together, each of the above factors may reasonably explain why Arab participants seemed underperformed their Jewish counterparts in the present experiment.

To summarize, results from Experiment 1 suggest that, contrary to a widely held theory (e.g., Gutchess, Welsh, Boduroglu, \& Park, 2006; Ji, Zhang, \& Nisbett, 2004; Wang \& Ross, 2005), culture per se does not markedly foster propensities toward reliance on particular memory strategies. Rather, the efficiency with which information is retained seems to be determined by the way it is structured/presented at its initial encounter. Although presenting information in a structured way appears to be beneficial in general, some structures (thematic structure) seem to enhance the retention of order information earlier than others (taxonomic structure) (Miller \& Eilam, 2008). The fact that such improvement was not found to interact with cultural background or presentation mode strongly suggests that it is inherently linked to the individual's maturation rather than being dependent on characteristics of the processed information or of the individual's cultural experience.

Jewish participants outperformed Arab participants - a finding we assume to reflect disadvantages originating from the complexity of Arabic orthography and its diglossic nature (Ibrahim, 2010; Ibrahim \& Aharon-Peretz, 2005; Ibrahim, Eviatar, \& Aharon-Peretz, 2002). Given that the factors underlying their weakness are not due to change, marked differences between Jewish and Arabs students in reading-based tasks are rather expected (Miller \& Peleg, 2010).

\section{EXPERIMENT 2: CONCEPT ORGANIZATION PARADIGM}

Experiment 2 aimed to reveal whether individuals apply existing knowledge prestructured by their cultural orientation and formal education to the active organization of information. To test this assumption, participants were asked to organize randomly distributed written word concepts into clusters.

\subsection{Method}

\subsubsection{Participants}

The participants tested in Experiment 2 were the same as those examined in Experiment 1 (Table 1).

\subsubsection{Material and stimuli}

Stimuli were six word lists comprised of nine familiar words. The preparation of the lists was based on the same criteria used in the STM experiment. Thus, the items of a list could be readily assembled taxonomically or thematically, or they could be organized by participants according to different, self-invented criteria.

The nine items in any given list were presented simultaneously and decontextualized (Table 6) as "draggable" objects at the top of a computer display. The participants' were asked to drag them from the list and organize them into clusters according to their own criteria.

Table 6. Example of a nine-word list in Arabic and Hebrew used in the concept organization task

\begin{tabular}{|c|c|c|c|c|c|c|}
\hline Language & Item2 & Item3 & Item6 & Item7 & Item8 & Item9 \\
\hline Arabic & حلو لفل & أخضر & توت & مر & أحمر & زيتون \\
\hline Hebrew & פלפל & ירוק & תות & מריר & אדום & זיתים \\
\hline English & pepper & Green & strawberry & bitter & red & olive \\
\hline
\end{tabular}

Participants completed the concept organization paradigm always after the STM paradigm with instructions given in their native tongue. The experimenter informed them that they would see a list of words displayed on the top of the computer display and initiated the display of the first. She then told the participants: "Your task is to group these words into word clusters in a way that makes sense to you. To do this, simply drag the words into two or more groups."

The six word lists were presented one after another, with each new list displayed only after participants informed the experimenter that they were satisfied with how they had grouped the concepts of a particular list. Following the completion of each list, the experimenter asked participants what clustering strategies they had used to group the word concepts and jotted down their answers on a protocol sheet. After the words of the last list were grouped, the experimenter saved the products of participants for subsequent analysis.

\subsection{Results}

The main dependent variable measured in this experiment was type of concept organization. This was defined as the participant's propensity to use a particular criterion to cluster the word concepts. A qualitative examination of the participants' concept clusters revealed three optional organization strategies (Figure 1 - 3): a) taxonomic - participants 
grouped word concepts entirely or predominantly according to a taxonomic relationship (with the remainder left unrelated); b) thematic - participants grouped them entirely or predominantly according to a thematic relationship (with the remainder left unrelated); and (c) mixed - participants grouped the concepts into several clusters, partly according to a taxonomic relationship and partly according to a thematic one, or they grouped them all (according to both a taxonomic and a thematic relationship) into a single cluster.
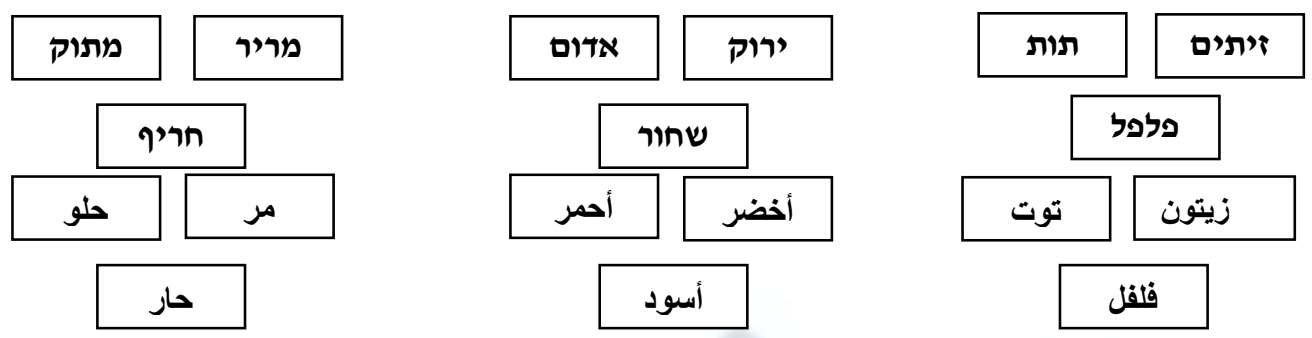

Figure 1: Example, in Hebrew and Arabic, of a taxonomic cluster

Note. English from left to right: sweet, bitter, spicy / red, green, black / strawberry, olive, pepper
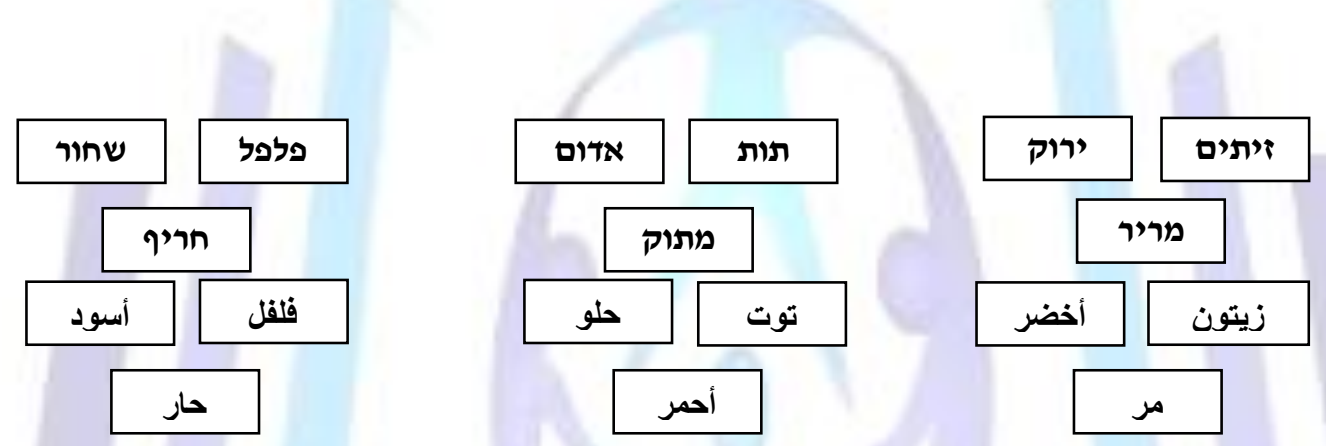

Figure 2: Example, in Hebrew and Arabic, of a thematic cluster

Note. English from left to right: black, pepper, spicy / red, strawberry, sweet / green, olive, bitter

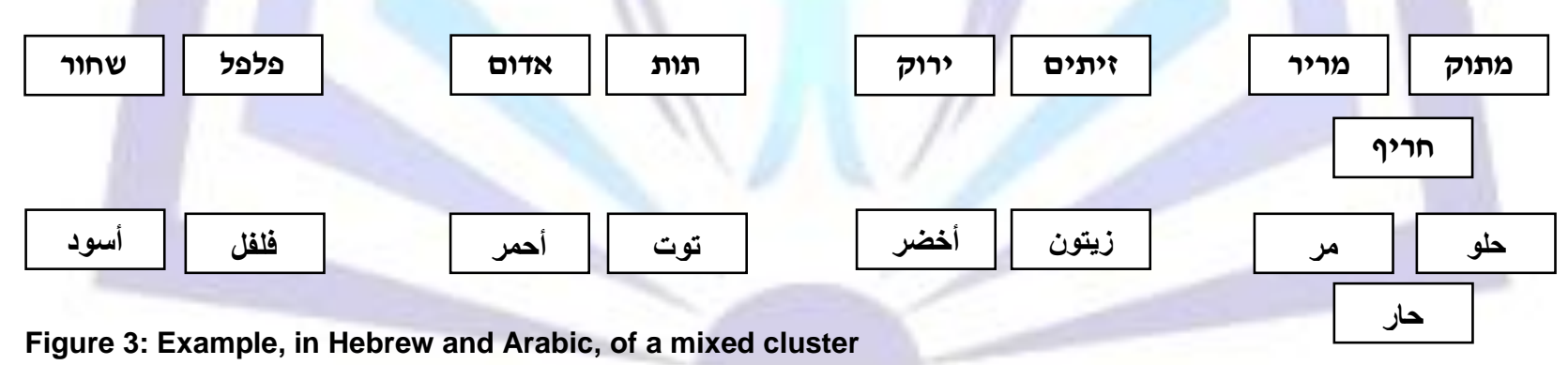

Note. English from left to right: black, pepper / red, strawberry / green, olive / bitter, sweet, spicy

We calculated the participants' reliance on each of the three identified grouping strategies as a percentile. In instances in which participants produced ambiguous concept clusters, we referred to their own verbatim explanations to allow adequate coding. Percentile means for reliance on the three concept organization strategies, with reference to participants' cultural background and grade-level, are presented in Table 7.

We compared reliance of the three concept organization strategies by means of ANOVA, computing type of concept organization (taxonomic, thematic, mixed) as a within-subject factor and cultural background and grade-level as two between-subject factors. Type of concept organization produced a statistically significant main effect, $F(2,114)=71.73, p$ $<.001, \eta^{2}=.39$, suggesting that, overall, participants tended to use some of the organization strategies more often than others. 
Table 7. Percentile group means for reliance on the three concept organization strategies (standard deviations in parentheses)

\begin{tabular}{lcccc}
\hline Presentation Mode & Elementary School & High School & University & All Levels \\
\hline \multirow{4}{*}{ Thematic } & Jewish Participants - Individualist Background & & $53 \%(41)$ \\
Taxonomic & $34 \%(38)$ & $68 \%(36)$ & $57 \%(44)$ & $32 \%(37)$ \\
Mixed & $50 \%(36)$ & $24 \%(35)$ & $22 \%(36)$ & $15 \%(26)$
\end{tabular}

\begin{tabular}{|c|c|c|c|c|}
\hline \multicolumn{5}{|c|}{ Arab Participants - Collectivist Background } \\
\hline Thematic & $89 \%(16)$ & $78 \%(32)$ & $69 \%(38)$ & $78 \%(31)$ \\
\hline Taxonomic & $8 \%(14)$ & $7 \%(19)$ & $21 \%(39)$ & $12 \%(27)$ \\
\hline Mixed & $3 \%(9)$ & $15 \%(26)$ & $10 \%(15)$ & $10 \%(18)$ \\
\hline \multicolumn{5}{|c|}{ All Participants } \\
\hline Thematic & $62 \%(40)$ & $73 \%(34)$ & $63 \%(41)$ & $66 \%(39)$ \\
\hline Taxonomic & $29 \%(35)$ & $15 \%(29)$ & $22 \%(37)$ & $22 \%(34)$ \\
\hline Mixed & $9 \%(18)$ & $12 \%(20)$ & $15 \%(27)$ & $12 \%(22)$ \\
\hline
\end{tabular}

The type of concept organization $\times$ grade-level interaction was statistically not significant, $F(4,114)=1.30$, implying that, overall, formal education did not modify participants' inclination to rely on certain concept organization strategies more than others. Of note, however, type of concept organization significantly interacted with cultural background, $F(2$, $114)=12.40, p<.001, \eta^{2}=.10$, suggesting culture to bias the participants' concept organization strategy. A statistically significant cultural background $\mathrm{x}$ grade-level $\mathrm{x}$ type of concept organization interaction, $F(4,114)=4.40, p<.01, \eta^{2}=.07$, implied that culture-related bias was further modified by participants' level of schooling (Table 7)

We conducted three paired $t$-test post-hoc comparisons (one-tailed) to understand the participants' concept organization preferences and the way these are modified by their cultural background and grade-level. The first of them contrasted the percentile use of the three concept organization strategies, overall. Results indicated that, on the whole, participants tended to cluster concepts thematically more often than taxonomically, $t(119)=6.94, p<.001$, or mixed, $t(119)=-10.98, p$ $<.001$. In addition, they clustered the word concepts significantly more often taxonomically than according to a mixed strategy, $t(119)=-2.48, p<.05$.

A second line of post hoc analysis clarified the final significance of the interaction between the participants' cultural background and their propensity towards the use of a particular concept organization strategy. For this purpose we run three Oneway procedures comparing Arab and Jewish participants in terms of their reliance on any of the identified concept organization strategies. Arab participants, overall, grouped word concepts thematically significantly more often than their Jewish counterparts, $F(1,119)=15.01, p<.001$. In contrast, the application of a taxonomic organization strategy was found to be more prominent among the Jewish participants, $F(1,119)=12.12, p=.001$. Finally, reliance on a mixed organization strategy did not distinguish between the two groups, $F(1,119)=1.69$.

A third line of post hoc analysis was conducted to clarify the three-way interaction of cultural background $x$ grade-level $x$ type of concept organization. We examined differences in reliance on organization strategies by Jewish and Arab participants at each of the grade-levels via a series of paired $t$-tests. Arab participants, at all three levels of education, organized words according to a thematic strategy significantly more often than according to a taxonomic or mixed strategy (all $p$ 's $\leq .01$ ). No significant differences were found between the application of a taxonomic organization strategy and a mixed one. The analyses revealed a more complex picture for Jewish participants. At the elementary school level, they organized the stimulus materials according to taxonomic criteria significantly more often than mixed criteria $(p<.01)$. In contrast, no statistically significant evidence was found suggesting that a taxonomic organization strategy was used more often than a thematic one. Nor was there evidence that thematic clustering was used more frequently than mixed clustering. At the high school and university levels, Jewish participants - like their Arab counterparts - preferred thematic organization over taxonomic or mixed organization (all $p$ 's <.05). There was no evidence of preference for taxonomic over mixed organization at these higher levels of schooling.

In the last line of post hoc analysis, we directly compared Jewish and Arab students' reliance on each organization strategy per grade-level with Oneway analyses of variance. At the elementary school level, the products of Arab participants tended to reflect a markedly higher percentage of thematic concept organization than those of their Jewish counterparts, $F(1,39)=35.83, p<.001$. In contrast, the inclination of Jewish participants to rely on taxonomic organization criteria was found to be significantly higher than for Arab participants, $F(1,39)=24.48, p<.001$. Mixed grouping did not distinguish between Jewish and Arab participants, $F(1,39)=5.01, p<.05$. Analyses for high school and university levels failed to disclose further significant differences between these groups. However, at the high school level, the use of a taxonomic strategy tended to be somewhat more - although not significantly more - pronounced among Jewish participants, $F(1,39)=3.82$. 


\subsection{Discussion}

In contrast to the STM task, which required passive perception of information, the concept organization paradigm was designed to examine participants' propensity to use specific strategies while actively pulling together decontextualized and randomly distributed familiar words into clusters. In line with a position that considers information processing to be biased by cultural preferences (e.g., Masuda \& Nisbett, 2001; Ji, Zhang \& Nisbett, 2004; Gutchess, Yoon, Luo, et al., 2006 MacGregor \& Godfrey, 2011), we expected Jewish participants (individualist culture) to group the word stimuli predominantly according to taxonomic clustering criteria and Arab participants (collectivist culture) according to thematic clustering criterion (Hypothesis 3). We further assumed that such cultural predisposition towards reliance on particular organization strategies would be more prominent as individuals gain more schooling (Hypothesis 4). Findings provide only partial support for the validity of these hypotheses.

Unexpectedly, participants from both cultural backgrounds - in particular at the more advanced levels of education prefer thematic over taxonomic organization (Table 7). This general tendency toward the use of thematic organization diverges from findings reported by the majority of studies (e.g., Denney \& Ziobrowski, 1972; Markman \& Hutchinson, 1984; Masuda \& Nisbett, 2001; Nisbett, Peng, Choi, \& Norenzayan, 2001). It does, however, line up with findings suggesting that thematic relations may be preferred over other complex knowledge structures at all grade-levels (e.g., Miller \& Eilam, 2008).

It is noteworthy that Jewish elementary school students did not manifest a significant preference for a thematic clustering strategy, as opposed to a taxonomic one, whereas those Jewish participants at more advanced levels of education did. Moreover, in contrast to their Jewish counterparts, Arab participants seemed to prefer reliance on thematic knowledge from the earliest age tested in this experiment. It is of course tempting to interpret such evidence as indicative of the existence of a basic dispositional discrepancy between these two cultures (e.g., Gutchess, Yoon, Luo, et al., 2006; Ji, Zhang, \& Nisbett, 2004; MacGregor \& Godfrey, 2011). However, a closer look at the findings within the context of the rest of the evidence points to the dangers of oversimplifying the matter.

First, Arab participants indeed tended to organize the word concept lists more thematically than taxonomically. However, the same was also true for Jewish participants above the elementary school level, although in a somewhat more moderate form. Second, contrary to the expectation that increased exposure to a culture magnifies individuals' cultural disposition, Jewish participants, having manifested a propensity to organize information taxonomically at the elementary school level, switched to a predominantly thematic concept organization strategy at the more advanced levels of education. Finally, there was no statistically significant evidence that distinguished Jewish and Arab participants above the elementary school level, neither with regard to reliance on a thematic organization strategy nor with regard to any of the other organization strategies. Taken together, such evidence challenges the assumption that culture per se markedly determines the way individuals process information. It also seems to contradict the theory that postulates a shift from the use of thematic organization to more complex organization strategies in the course of maturation (Blewitt, 1994; Denney \& Ziobrowski, 1972; Inhelder \& Piaget, 1964; Kofsky, 1966; Lowell, 1980).

Jewish - but not Arab - participants manifested some reliance on taxonomic knowledge in order to organize the word concepts. Why such a dispositional discrepancy was manifested at an early age but disappeared at more advanced ones remains an open question. The finding is particularly puzzling if one assumes that cultural preferences become more salient as individuals grow older and that younger children may have a natural tendency to favor thematic relations over other complex relations, such as taxonomic ones (Borghi \& Caramelli, 2003; Denney \& Ziobrowski, 1972; Inhelder \& Piaget, 1964; Markman \& Hutchinson, 1984). A possibility worthy of consideration in this regard is that teachers in Arab and Jewish schools may encourage their students to rely on particular organization strategies more than others. For example, it may well be that the explicit learning of categories at early grade-levels is more pronounced in Jewish schools than it is in Arab schools. If corroborated by future research, the enhanced disposition of Jewish elementary school students to organize word concepts according to taxonomic criteria would make sense.

In summary, findings revealed from the concept organization task suggest that - if required to manipulate (e.g., structure) information - individuals are likely to rely on knowledge that reflects real-life experience rather than knowledge of artificial cognitive models taught in school. Overall, participants - regardless of their cultural background - favored thematic organization over taxonomic. It may, of course, be argued that, at the elementary school level, this tendency was more prominent among Arab students than Jewish ones. Whether the origin of such a discrepancy reflects a true cultural disposition remains, however, questionable until verified by future research. The fact that such a discrepancy was restricted to an educational level at which categories are explicitly taught as a part of the curriculum actually points to variance in emphasis on the development of categorical knowledge rather than true cultural preferences as the underlying cause.

\section{GENERAL DISCUSSION}

The goal of the two experiments was to examine whether cultural background and formal education bias the way individuals process information in two different contexts - one that required the retention and recall of prestructured information in a primarily passive manner and another asking for active application of structural knowledge to organize randomly presented word concepts. We expected performance in both tasks to be biased by peculiarities inherent to individuals' knowledge structures, peculiarities we assumed to reflect (among others) cultural and educational preferences. 
Evidence obtained from both experiments warrants several conclusions that call for reconsideration of at least some widely held positions in the field. First, rather than cultural or educational stimulations, it is the way information is prestructured at encounter that seems to exert a fundamental influence on how efficiently it is processed by individuals (Poirier, Dhir, Saint-Aubin, Tehan, \& Hampton, 2011; Poirier \& Saint-Aubin, 1996; Vicari, Pasqualetti, Marotta, \& Carlesimo, 1999). Our findings explicitly demonstrate the power inherent in organization for enhancing the perception and orderly retention of information (Miller \& Eilam, 2008). They further suggest that individuals approach information with various types of prestructured knowledge simultaneously (Imai, Saalbach, \& Stern, 2010; Waxman \& Gelman, 1986). Part of this knowledge seems to depict real-life experience, while part seems to reflect abstract mental models, such as categories, that are explicitly taught in class. It is noteworthy, however, that even if the latter may impact information processing at the time they are explicitly introduced to students, they do not seem to become the dominant vehicle of approach to the organization of newly encountered information. Rather, more intuitive information processing strategies seem to resume dominance once the application of such artificial models to the organization of information ceases to be explicitly emphasized in the course of formal instruction.

Participants' knowledge seems to enhance the assimilation and retention of information structured in essentially different ways (Waxman \& Gelman, 1986). It is noteworthy that - beyond enhancements originating from structuring to-be-recalled information - neither the Jewish nor the Arab participants' recall reflected bias according to assumed cultural dispositions or educational stimulation (Imai, Saalbach \& Stern, 2010). It may, of course, be argued that the influence of cultural preferences on information processing may not manifest in instances in which information is not manipulated by the processor, as was the case in the STM task. Tasks in which individuals are asked to impose structure on information - as in our concept organization task - may therefore be more suitable for revealing the influence of culture and education. Participants' propensity to organize thematically and not taxonomically, particularly above the elementary school level regardless of cultural background - challenges the claim that culture notably biases the way individuals process information (e.g., Gutchess, Yoon, Luo, et al., 2006; Ji, Zhang, \& Nisbett, 2004). This conclusion is also warranted given that neither in the STM task nor in the concept organization task did an increase in participants' level of education magnify potential cultural bias on performance.

While culture was not found to markedly influence task performance in the two experiments, some evidence from the concept organization task - namely, the enhanced use of a taxonomic organization strategy by Jewish participants seems to suggest that education may have impacted performance to some extent. Interestingly, however, instead of becoming more pronounced over time, such educational gains were not durable in the long term. Moreover, there was no evidence that it was paralleled in Arab participants, probably because the teaching of categorical knowledge is not as emphasized in their curriculum as it is in that of their Jewish counterparts. Both these findings also challenge the postulation of a maturation-related thematic-to-taxonomic shift in individuals' disposition to approach information (Borghi \& Caramelli, 2003; Lin \& Murphy, 2001).

Because thematic knowledge reflects relations between events (pieces of information) as they are experienced, it is more comprehensible to individuals as they approach the processing of information. Moreover, because it reflects the way people experience reality, it may actually prove most effective for optimal adaptation to one's surroundings, so that its application is continuously reinforced. More abstract organization forms, such as taxonomic structures, are superficial and primarily taught rather than experienced. Consequently, they are not intuitive and are hardly ever used to deal with situations in daily life. Thus, fostering the application of such knowledge/strategies may in fact require greater emphasis at all grade-levels in order to disclose their relevance for understanding the world. If successful, such an effort could definitely lead to enrichment of habitual ways of thinking about events and, in the long term, to improved achievement in specific domains.

The unexpected performance cluster of the youngest Jewish participants may therefore warrant some reconsideration. As mentioned earlier, children from a Jewish background may have brought more and deeper internalized taxonomy-based information processing strategies to the concept organization task. Provided this to be true, when asked to organize information at a time they are explicitly taught categories in class, this knowledge may well compete with more intuitive information strategies, such as thematic ones. In Arab schools, where - perhaps due to cultural preferences - the curriculum places less emphasis on the explicit teaching of taxonomies, more natural organization strategies may continue to dominate information processing. This would explain why Arab elementary school students did not manifest the same enhanced propensity to rely on categorical knowledge for the organization of word concepts as did their Jewish counterparts.

It is of interest that, even though the group of Jewish elementary school students manifested markedly enhanced reliance on taxonomic knowledge to organize the word concepts, this was not their sole strategy; thematic organization continued to play a statistically comparable role in their attempts to impose structure on randomly presented concepts. Moreover, contrary to expectations, at more advanced levels of education, Jewish participants exhibited a clear tendency towards the use of thematic relation knowledge for this purpose. The reason for this apparent retreat to a less abstract information processing strategy remains puzzling in many regards and requires further exploration. However, the sheer fact that such a retreat occurred seems to suggest that we prefer to process information in ways that link it to real-life experience rather than abstract and often artificial cognitive models. More specifically, repeated explicit exposure to such models via formal instruction may indeed lead individuals to temporarily apply them to information they are asked to process. However, in the long term, their more intuitive strategies seem to dominate their approach to information, with a thematic strategy undoubtedly being one of them 
Mixed clustering was, overall, the least used grouping strategy. This suggests that individuals tended to approach concept organization in our experiment with one and the same organization strategy, rather than concurrently using different strategies. Whether this reflects a cultural and educational failure to promote cognitive flexibility, a biological factor or a task-inherent constraint needs to be addressed in future research. Significantly, there was no notable change in participants' reliance on a mixed clustering strategy from one educational level to the next. In fact, mixed clustering remained far less common than thematic clustering for all groups. These findings strongly suggest that participants' tendency to rely on thematic knowledge to group the word concepts was not transitional in nature; rather, it appears to reflect their natural propensity to use knowledge structures that have evolved from real-life experience in order to interpret the meaning of perceived information.

In summary, individuals' knowledge seems to be structured in ways that enhance the assimilation of new information with compatible structures. In instances where they encounter information in unstructured form, they seem to manifest a marked propensity towards its organization in ways that reflect the relationships between phenomena as they are experienced in real life. While enhanced educational emphasis may temporarily lead to the use of less intuitive information processing strategies, such strategies seem to be abandoned once they are not longer explicitly taught. Awareness of this propensity may help teachers enhance their students' learning and understanding of general and domain-specific curriculum contents by overtly linking them to or contrasting them with their real-life experience.

\section{CONCLUSIONS, LIMITATIONS AND FUTURE RESEARCH}

Knowledge seems to be structured in ways that enhance the assimilation of new information with compatible structures. In instances individuals encounter information in unstructured form, they seem to organize it in ways that reflect the relationships between phenomena as they are experienced in real life. The influence of culture on how individuals process information seems to be marginal and overshadowed by other more dominant factors, such as the structure of the processed information at encounter.

The majority of Arab participants tested were residents of a city, Haifa, with a mixed, yet predominantly Jewish population. Moreover, Jewish participants were descendants of immigrants who came to Israel from various countries with multiple cultures (i.e., Ashkenazi Jews or Oriental Jews). Thus, both Arab and Jewish participants may not have been pure representatives of one specific culture. This possibility should be accounted for in designing future research with the goal to substantiate and expand findings from the present study.

\section{REFERENCES}

1. Anderson, J. R. (1991). The adaptive nature of human categorization. Psychological Review, 98, 409-429.

2. Barrouillet, P., Bernardin, S., Portrat, S., Vergauwe, E., \& Camos, V. (2007). Time and cognitive load in working memory. Journal of Experimental Psychology: Learning, Memory, and Cognition, 33, 570-585.

3. Borghi, A. M., \& Caramelli, N. (2003). Situation bounded conceptual organization in children: From action to spatial relations. Cognitive Development, 18, 49-60.

4. Carey, S. (1985). Conceptual change in childhood. Cambridge, MA: Bradford Books, MIT Press.

5. Cowan, N., Hismjatullina, A., AuBuchon, A. M., Saults, J. S., Horton, N., Leadbitter, K., \& Towse, J. (2010). With development, list recall includes more chunks, not just larger ones. Development Psychology, 46, 1119-1131.

6. Denney, N., \& Ziobrowski, M. (1972). Development changes in clustering criteria. Journal of Experimental Child Psychology, 13, 275-282.

7. Diab, K. \& Mi'ari, M. (2007). Collective identity and readiness for social relations with Jews among Palestinian Arab students at the David Yellin Teacher Training College in Israel. Intercultural Education, 18, 427-444.

8. Gelman, S. A. (1998). Categories in young children's thinking. Young Children, January, 20-26.

9. Gelman, S. A., \& Kalish, C. W. (2006). Conceptual development. In D. Kuhn \& R. Siegler (Eds.), Handbook of child psychology, Vol. 2: Cognition, perception, and language (pp. 687-733). New York: Wiley.

10. Greene, T. R. (1991). Text manipulations influence children's understanding of class inclusion hierarchies. Journal of Experimental Child Psychology, 52, 354-374.

11. Gutchess, A. H., \& Indeck, A. (2009). Cultural influences on memory. Progress in Brain Research, 178, 137-150.

12. Gutchess, A. H., Welsh, R. C., Boduroglu, A., \& Park, D. C. (2006). Cultural differences in neural function associated with object processing. Cognitive, Affective \& Behavioral Neuroscience, 6, 102-109.

13. Gutchess, A. H., Yoon, C., Luo, T., Feinberg, F., Hedden, T., Jing, Q., et al. (2006). Categorical organization in free recall across culture and age. Gerontology, 52, 314-323.

14. Hofstede, G. \& Bond, M. H. (1984). Hofstede's culture dimensions: An independent validation using Rokeach's value survey. Journal of Cross-Cultural Psychology, 15, 417-433. 
15. Ibrahim, R. (2010). Literacy problems in Arabic: Sensitivity to diglossia in tasks involving working memory. Journal of Neurolinguistics, doi:10.1016/j.jneuroling.2010.10.003

16. Ibrahim, R., \& Aharon-Peretz, J. (2005). Is literary Arabic a second language for native Arab speakers? Evidence from a semantic priming study. The Journal of Psycholinguistic Research, 34, 51-70.

17. Ibrahim, R., Eviatar, Z., \& Aharon-Peretz, J. (2002). The characteristics of Arabic orthography slow its processing. Neuropsychology, 16, 322-326.

18. Imai, M., Saalbach, H., \& Stern, E. (2010). Are Chinese and German children taxonomic, thematic or shape biased? Influence of classifiers and cultural contexts. Frontiers in Psychology, 1, 194. doi:10.3389/fpsyg.2010.00194

19. Inhelder, B., \& Piaget, J. (1964). The early growth of logic in the child. New York: Norton.

20. Ji, L. J., Zhang, Z., \& Nisbett, R. E. (2004). Is it culture or is it language? Examination of language effects in crosscultural research on categorization. Journal of Personality and Social Psychology, 87, 57-65.

21. Kalish, C. W., \& Gelman, S. A. (1992). On wooden pillows: Multiple classification and children's category-based inductions. Child Development, 63(6), 1536-1557.

22. Khattab, N. (2003). Explaining educational aspirations of minority students: The role of social capital and students' perceptions. Social Psychology of Education, 6, 283-302.

23. Kitayama, S., Duffy, S., Kawamura, T., \& Larsen, J. (2003). Perceiving an object and its context in different cultures: A cultural look at New Look. Psychological Science, 14, 201-206.

24. Klausmeier, J. H. (1980). Learning and teaching concepts: A strategy for testing applications of theory. New York: Academic Press.

25. Kofsky, E. (1966). A scalogram study of classificatory development. Child Development, 37, 291-294.

26. Koutstaal, W., Wagner, A.D., Rotte, M., Maril, A., Buckner, R.L., \& Schacter, D.L. (2001). Perceptual specificity in visual objest priming: functional magnetic resonance imaging evidence for a laterality difference in fusiform cortex. Neuropsychologia, 39, 184-199.

27. Lin, E. L., \& Murphy, G. L. (2001). Thematic relations in adults' concepts. Journal of Experimental Psychology: General, 130(1), 3-28.

28. Lowell, W. E. (1980). The development of hierarchical classification skills in science. Journal of Research in Science Teaching, 17, 425-433.

29. MacGregor, D. G., \& Godfrey, J. (2011). Arab cultural influences on intertemporal reasoning. Social Science Research Network. Available at http://ssrn.com/abstract=1966639 or http://dx.doi.org/10.2139/ssrn.1966639.

30. Mahajna, A., Benzion, U., Bogaire, R., \& Shavit, T. (2007). Subjective discount rates among Israeli Arabs and Israeli Jews. Journal of Socio-Economics, 37, 2513-2522.

31. Markman, E. M. (1989). Categorization and naming in children: Problems of induction. Cambridge, MA: MIT Press.

32. Markman, E. M., \& Hutchinson, J. E. (1984). Children's sensitivity to constraints on word meaning: Taxonomic versus thematic relations. Cognitive Psychology, 16, 1-27.

33. Masuda, T., \& Nisbett, R. E. (2001). Attending holistically versus analytically: Comparing the context sensitivity of Japanese and Americans. Journal of Personality and Social Psychology, 81, 922-934.

34. Medin, D. L., \& Atran, S. (2004). The native mind: Biological_categorization_and reasoning in development and across_cultures. Psychological Review, 111(4), 960-983.

35. Medin, D. L., Coley, J. D., Storms, G., \& Hayes, B. K. (2003). A relevance theory of induction. Psychonomic Bulletin \& Review, 10, 517-532.

36. Micklo, S. J. (1995). Developing young children's classification and logical thinking skills. Childhood Education, Fall, 24-28.

37. Mikulicer, M., Weller, A., \& Florian, V. (1993). Sense of closeness to parents and family rulers: A study of Arab and Jewish youth in Israel. International Journal of Psychology, 28, 23-35.

38. Miller, P., \& Eilam, B. (2008). Development in the thematic and containment-relation-oriented organization of word concepts. Journal of Educational Research, 101, 350-362.

39. Miller, P., \& Peleg, O. (2010). Doomed to read in a second language: Implications for learning. Journal of Psycholinguistic Research, 39, 51-65.

40. Murphy, G. L. (2002). The big book of concepts. Cambridge, MA: MIT Press.

41. Nisbett, R. E., Peng, K., Choi, I., \& Norenzayan, A. (2001). Culture and systems of thought: Holistic versus analytic cognition. Psychological Review, 108, 291-310. 
42. Nguyen, S. P., \& Murphy, G. L. (2003). An apple is more than just a fruit: Cross-classification in children's concepts._Child_Development, 74(6), 1783-1806.

43. Pennequin, V., Fontaine, R., Bonthoux, F., Scheuner, N., \& Blaye, A. (2006). Categorization deficit in old age: Reality or artefact? Journal of Adult Development, 13, 1-9.

44. Poirier, M., Dhir, P., Saint-Aubin, J., Tehan, G., \& Hampton, J. (2011). The influence of semantic memory on verbal short-term memory. In B. Kokinov, A. Karmiloff-Smith, \& N. J. Nersessian (eds.), European perspectives on cognitive science. Sofia: New Bulgarian University Press.

45. Poirier, M., \& Saint-Aubin, J. (1995). Memory for related and unrelated words: Further evidence on the influence of semantic factors in immediate serial recall. The Quarterly Journal of Experimental Psychology, 48, 384-404.

46. Poirier, M., \& Saint-Aubin, J. (1996). Immediate serial recall, word frequency, item identity and item position. Canadian Journal of Experimental Psychology, 50, 408-412.

47. Poirier, M., \& Saint-Aubin, J. (1999). Semantic similarity and immediate serial recall: Is there a detrimental effect on order information? The Quarterly Journal of Experimental Psychology, 52, 367-394.

48. Resh, N., \& Benavot, A. (2002). What is actually being taught in schools? Diversity and uniformity in the implementation of the official curriculum in Israeli junior high schools. Jerusalem: The NCJW Research Institution for Innovation in Education, Hebrew University (Hebrew).

49. Sabbagh, C., Faher-Aladeen, R., \& Resh, N. (2004). Evaluation of grade distributions: A comparison of Jewish and Druze students in Israel. Social Psychology of Education, 7, 313-338.

50. Sagy, S., Orr, E., Bar-On, D. \& Awwad, E. (2001). Individualism and collectivism in two conflicted societies: Comparing Israeli-Jewish and Palestinian-Arab high school students. Youth and Society, 32, 3-30.

51. Swirski, S., \& Dagan-Buzaglo, N. (2009). Separation, inequality and faltering leadership: Public education in Israel. Tel Aviv: Adva Center, Information on Equality and Social Justice in Israel (Hebrew).

52. Triandis. H. C. (1995). Individualism and collectivism. San Francisco: Westview Press.

53. Tse, C.S. (2009). The role of associative strength in the semantic relatedness effect on immediate serial recall. Memory, 17, 874-891.

54. Vicari, S., Pasqualetti, P., Marotta, L., \& Carlesimo, GA. (1999). Word list learning in normally developing children: Effects of semantic organization and retention interval. Italian Journal of Neurological Sciences, 20, 119-128.

55. Wang, Q., \& Ross, M. (2005). What we remember and what we tell: The effects of culture and self-priming on memory representations and narratives. Memory, 13, 594-606.

56. Wang, Q., \& Ross, M. (2010). Why we remember and what we remember: Culture and autobiographical memory. Perspectives on Psychological Science, 5, 401-409.

57. Waxman, S., \& Gelman, R. (1986). Preschoolers' use of superordinate relations in classification and language. Cognitive Development, 1, 139-156.

\section{Authors' biography}

Paul miller is the head of Department of special education at University of Haifa, where he is an Associate Professor of Psycholinguistics. His activity as researcher and lecturer is related to the cognitive functioning of student with prelingual deafness, students with learning disabilities, and regular students. In particular, I'm interested in how individuals' primary language impacts their processing of information (thinking).

Nancy Joubran-Awadie is graduate student in the Master's Program of the University of Haifa's Department of Learning Disabilities. Her interests are in the development of cognitive and reading ability among Arabic speaking children.

Raphiq Ibrahim is a senior researcher at the Edmond J. Safra Brain Research Center and the Department of Learning Disabilities at University of Haifa, where he is an Associate Professor of Neuropsychology. His research focuses on the cognitive processing of oral and written language. $\mathrm{He}$ is investigating brain regions involved in monolinguals and language selection processes in bilinguals and focuses on the hemispheric specialization of higher cognitive functions. 